\title{
WASSERSTEIN DISTANCE ON CONFIGURATION SPACE
}

\author{
L. DECREUSEFOND
}

\begin{abstract}
We investigate here the optimal transportation problem on configuration space for the quadratic cost. It is shown that, as usual, provided that the corresponding Wasserstein is finite, there exists one unique optimal measure and that this measure is supported by the graph of the derivative (in the sense of the Malliavin calculus) of a "concave" (in a sense to be defined below) function. For finite point processes, we give a necessary and sufficient condition for the Wasserstein distance to be finite.
\end{abstract}

\section{INTRODUCTION}

The optimal transportation problem stems back to the eighteenth century when G. Monge addressed the optimal way to move earth particles from one location to another. It is only in the forties of the last century that Kantorovitch gave this problem its modern form and a complete solution. According to the Kantorovitch approach, the optimal transportation problem or Monge-Kantorovitch problem (MKP for short) reads as follows: given two probability measures $\mu$ and $\nu$ on a Polish space $X$ and a cost function $c$ on $X \times X$, does there exist a probability measure $\gamma$ on $X \times X$ which minimizes $\int c \mathrm{~d} \beta$ among all probability measures $\beta$ on $X \times X$ with first (respectively second) marginal $\mu$ (respectively $\nu$ ) ? One can furthermore ask whether the optimal measure is unique and which properties it has. So far, the mainly investigated situations suppose that $X=\mathbf{R}^{n}$ or a finite-dimensional manifold with a cost function which is $c(x, y)=h(|x-y|)$ where $h$ is a convex (or concave) function on $\mathbf{R}$.

Varying cost functions and underlying spaces yields to numerous interesting inequalities often with optimal constants (see [Vil03 and references therein) or to new insights on known theorems such as Strassen Theorem about stochastic ordering (see [RR98a, RR98b]). Moreover, in a large part of investigated cases, the optimal measure is unique and is supported by the graph of a function $T$, i.e., $\gamma=(\operatorname{Id} \otimes T)^{*} \mu$. This map $T$ gives raise to a coupling, said optimal, between the measure $\mu$ and $\nu$ : If $A$ is a r.v. distributed according to $\mu$ then $T(A)$ is distributed according to $\nu$ and this construction of the two distributions on the same probability space is optimal in the sense that it minimizes $E[c(A, B)]$ among all the r.v. $B$ distributed according to $\nu$. Optimal coupling is a well known tool to obtain inequalities between random variables (see [Tho00]) but to the best of our knowledge, the optimal

1991 Mathematics Subject Classification. 60B05, 60H07, 60G55.

Key words and phrases. Configuration space, Monge-Kantorovitch, Optimal transportation problem, Poisson process. 
coupling has to be explicitly built to obtain these bounds. Meanwhile, optimal transportation theory in the solved cases, indicates that the optimal coupling (or transportation) map can be written as the graph of a "concave" function, so independently of the precise description of the map, one can obtain interesting inequalities just knowing this property of the optimal map.

Our goal is here to develop the theory of optimal transportation for point processes (or configuration spaces) with the objective to obtain a machinery yielding inequalities similar to those obtained by the Stein's method [BHJ92, BM02, BC01, BX00.

The first step is to define a cost between configurations. Several possibilities can be envisioned, we chose here a cost with a strong physical interpretation: given two configurations (or sets of points), $\left(x_{1}, \cdots, x_{m}\right)$ and $\left(y_{1}, \cdots, y_{m}\right)$, the cost is roughly defined as $\inf _{\sigma \in m} \sum_{j}\left|x_{j}-y_{\sigma(j)}\right|^{2}$ where $m$ is the group of permutations over $\{1, \cdots, m\}$ (see 3 for the precise definition). The point is then to determine the cost to go from a configuration with $m$ points to a configuration with $m^{\prime}$ points, when $m \neq m^{\prime}$. In order to keep a physical meaning to the definition of our cost, it seems sensitive to impose an infinite value to something which is impossible. The negative consequence of this choice is that severe constraints are imposed (see Theorem 4.2) on two finite point processes for their Wasserstein distance to be finite. On the other hand, the positive consequence is that the optimal measure has a well defined structure. These constraints disappear when we deal with locally finite but not finite point processes and we still have a rigid structure for the optimal measure. It turns out that proving here the uniqueness and describing the form of the optimal measure is highly similar to the proof of the same properties for the Wiener space (see [FÜ04]).

This paper is organized as follows : we describe the Monge-Kantorovitch Problem in its general settings for a generic cost function on a product of two abstract Polish spaces, since we will need to instantiate these general results to different particular situations in the subsequent sections. Section 3 is devoted to general properties of the Wasserstein distance on configuration spaces irrespective to the properties of finiteness of the considered point processes. In Section 4, we work under the assumption that only a finite number of atoms are random in the $\mu$-configurations and we slightly modify our cost function to simultaneously solve optimal transportation between finite point processes and pave the way to the analysis for locally finite point processes. This latter analysis is done in Section 5 .

\section{Preliminaries}

For $X$ and $Y$ two Polish spaces, for $\mu$ (respectively $\nu$ ) a probability measure on $X$ (respectively $Y$ ), $\Sigma(\mu, \nu)$ is the set of probability measures on $X \times Y$ whose first marginal is $\mu$ and second marginal is $\nu$. We also need to consider a lower semi continuous function $c$ from $X \times Y$ to $\mathbf{R}^{+}$. The Monge-Kantorovitch problem associated to $\mu, \nu$ and $c$, denoted by $\operatorname{MKP}(\mu$, $\nu, c)$ for short, consists in finding

$$
\inf _{\gamma \in \Sigma(\mu, \nu)} \int_{X \times Y} c(x, y) \mathrm{d} \gamma(x, y) .
$$


More precisely, since $X$ and $Y$ are Polish and $c$ is l.s.c., it is known from the general theory of optimal transportation, that there exists an optimal measure $\gamma \in \Sigma(\mu, \nu)$ and that the minimum coincides with

$$
\sup _{(F, G) \in \Phi_{c}}\left(\int_{X} F \mathrm{~d} \mu+\int_{Y} G \mathrm{~d} \nu\right)
$$

where $(F, G)$ belongs whenever $F \in L^{1}(\mathrm{~d} \mu), G \in L^{1}(\mathrm{~d} \nu)$ and $F(x)+G(y) \leq$ $c(x, y)$. We will denote by $\mathcal{T}_{c}(\mu, \nu)$ the value of the infimum in (1). Solving the Monge-Kantorovitch problem on Polish spaces, is then essentially proving the finiteness of (11) and the uniqueness of the optimal measure. For $X=$ $Y=\mathbf{R}^{k}$ and $c$ taken to be the square euclidean distance, we have the second moment condition: for $\mathcal{T}_{c}(\mu, \nu)$ to be finite it is sufficient that $\int\|x\|^{2} \mathrm{~d} \mu$ and $\int\|x\|^{2} \mathrm{~d} \nu$ are finite, where $\|x\|$ is the euclidean norm (see [Vil03]). For further reference, we denote by $\mathcal{T}_{e}$ (where $e$ stands for euclidean) this distance between probability measures on $\mathbf{R}^{k}$. If $\mu$ is the Gaussian measure on $\mathbf{R}^{k}$ and $\nu$ is absolutely continuous with respect to $\mu$ with Radon-Nikodym density $L$, it is sufficient that $L$ has a finite entropy, i.e., the $\mu$-expectation of $L \ln L$ is finite, for $\mathcal{T}_{e}(\mu, L \mu)$ to be finite. This criterion extends to the infinite dimensional setting where $X=Y$ is a Wiener space and $c(x, y)=2^{-1} \| x-$ $y \|_{H}^{2}$, where $H$ is the associated Cameron-Martin space (see [FÜ04]). In full generality, we know from RR98a that if there exist $F \in L^{1}(\mathrm{~d} \mu)$ and $G \in L^{1}(\mathrm{~d} \nu)$ such that $c(x, y) \leq F(x)+G(y)$, then $\mathcal{T}_{c}(\mu, \nu)$ is finite.

Once the finiteness of $\mathcal{T}_{c}$ is ensured, it remains to know whether the optimal measure is unique. For, it is essential to see that a measure $\gamma \in$ $\Sigma(\mu, \nu)$, is optimal if and only if its support is $c$-cyclically monotone (see [Lev99, Rüs96]) : for any $\left(\left(x_{i}, y_{i}\right), i=1, \cdots, m\right) \in(\operatorname{supp} \gamma)^{m}$, we have

$$
\sum_{i=1}^{m} c\left(x_{i}, y_{i}\right) \leq \sum_{i=1}^{m} c\left(x_{i}, y_{\sigma(i)}\right)
$$

for any $\sigma \in m$, the group of permutations over $\{1, \cdots, m\}$. Moreover, the support of any optimal measure is included in the $c$-super-gradient of a $c$ concave function: For $F: X \rightarrow \overline{\mathbf{R}}=\mathbf{R} \cup\{+\infty\}$, its $c$-super-gradient, $\partial^{c} F$, is the subset of $\Gamma_{X} \times \Gamma_{Y}$ of $(x, y)$ such that $c(x, y)<\infty$ and

$$
F(x)-F(z) \geq c(x, y)-c(z, y) \text {, for any } z \text { such that } F(z)<+\infty \text {. }
$$

The section at $x, \partial^{c} F(x)$, is the set $\left\{y \in Y,(x, y) \in \partial^{c} F\right\}$. A function $F: X \rightarrow \mathbf{R}$ is called c-concave if there exist a set index $I,\left(y_{i}, i \in I\right)$ a family of elements of $Y$ and $\left(a_{i}, i \in I\right)$ a family of real numbers such that

$$
F(x)=\inf _{i \in I}\left(c\left(x, y_{i}\right)+a_{i}\right) .
$$

If we prove that the $c$-super gradient of a $c$-concave function is single valued, we are done, i.e., we have proved the uniqueness of the optimal measure. Indeed, if $\partial^{c} F(x)$ is reduced to a singleton for $\mu$-a.s. any $x \in X$, this means that $\partial^{c} F(x)$ is closed and the selection theorem then induces that there exists a measurable map $T$ such that $(x, y)$ belongs to $\partial^{c} F$ if and only if $y=T(x)$. The uniqueness follows then from the following lemma which we borrow from FÜ04. 
Theorem 2.1 (See FÜ04]). Let $X$ and $Y$ be two Polish spaces and $c$ be a lower-semi-continuous function from $X \times Y$ to $\mathbf{R}^{+} \cup\{+\infty\}$. Consider the Monge-Kantorovitch problem associated to the marginals $\mu$ on $X, \nu$ on $Y$ and $c$. Assume that for any optimal measure $\gamma$, there exists a measurable map $T_{\gamma}$ such that supp $\gamma \subset\left\{\left(x, T_{\gamma}(x)\right), x \in\right.$ supp $\left.\mu\right\}$. Then, there exist a unique optimal measure $\gamma$ and a unique measurable map $T$ such that $\gamma=(\operatorname{Id} \otimes T)^{*} \mu$.

Proof. For any probability measure $\gamma$ on $X \times Y$, we denote by $J(\gamma)$ the integral of $c$ with respect to $\gamma$ :

$$
J(\gamma)=\int_{X \times Y} c(x, y) \mathrm{d} \gamma(x, y) .
$$

Assume that $\gamma_{1}$ and $\gamma_{2}$ are two different optimal measures. Since $J$ is linear with respect to $\gamma, \gamma_{0}=\left(\gamma_{1}+\gamma_{2}\right) / 2$ is also optimal. We denote by $T_{0}$ a map whose graph contains the support of $\gamma_{0}$. Furthermore, for $i=1,2, \gamma_{i}$ is absolutely continuous with respect to $\gamma_{0}$. We denote by $L_{i}$ the RadonNikodym derivative of $\gamma_{i}$ with respect to $\gamma_{0}$. For any $f \in \mathcal{C}_{b}(X)$, we have

$$
\begin{aligned}
\int_{X} f(x) \mathrm{d} \mu(x) & =\int_{X \times Y} f(x) \mathrm{d} \gamma_{i}(x, y) \\
& =\int_{X \times Y} f(x) L_{i}(x, y) \mathrm{d} \gamma_{0}(x, y) \\
& =\int_{X} f(x) L_{i}\left(x, T_{0}(x)\right) \mathrm{d} \mu(x) .
\end{aligned}
$$

Therefore, we must have $L_{i}\left(x, T_{0}(x)\right)=1 \mu$-a.s. or in other words, $L_{i}=1$ $\gamma_{0}$-a.s. for $i=1,2$. This means that $\gamma_{1}=\gamma_{2}$ and then the uniqueness of the optimal measure for $\operatorname{MKP}(\mu, \nu, c)$.

Assume now that there exist two maps $T_{1}$ and $T_{2}$ such that $\gamma=\left(\operatorname{Id} \otimes T_{i}\right)^{*} \mu$. This implies that for any $f \in \mathcal{C}_{b}(X), f \circ T_{1}=f \circ T_{2} \mu$-a.s. hence that $T_{1}=T_{2}$ $\mu$-almost surely.

The simplest way to prove that the $c$ super-gradient of a $c$-concave function is single-valued is to show that a $c$-concave is "differentiable" in some sense. That is why, we need to introduce a notion of gradient on configuration space. The notations are mainly those of AKR98. Let $\Gamma_{X}$ be the configuration space over a Polish space $X$, i.e.,

$$
\Gamma_{X}=\{\eta \subset X ; \eta \cap K \text { is a finite set for every compact } K \subset X\} .
$$

We identify $\eta \in \Gamma_{X}$ and the positive Radon measure $\sum_{x \in \eta} \varepsilon_{x}$. Throughout this paper, $\Gamma_{X}$ is endowed with the vague topology, i.e., the weakest topology such that for all $f \in \mathcal{C}_{0}$ (continuous with compact support on $X$ ), the maps

$$
\eta \mapsto \int f \mathrm{~d} \eta=\sum_{x \in \eta} f(x)
$$

are continuous. When $f$ is the indicator function of a subset $B$, we will use the shorter notation $\eta(B)$ to denote the integral of $\mathbf{1}_{B}$ with respect to $\eta$. We denote by $\mathcal{B}\left(\Gamma_{X}\right)$ the corresponding Borel $\sigma$-algebra.

The intensity measure of a probability measure $\mu$ on $\Gamma_{X}$, is denoted by $E_{\mu} \eta$ and defined by $\left(E_{\mu} \eta\right)(B)=E_{\mu}[\eta(B)]$, for any $B \in \mathcal{B}\left(\Gamma_{X}\right)$. We assume henceforth that $E_{\mu} \eta$ is a positive Radon measure on $\mathcal{B}\left(\Gamma_{X}\right)$. 
In what follows, we will take $X=\mathbf{R}^{k}$ for some $k \geq 1$. Let $V(X)$ be the set of $\mathcal{C}^{\infty}$ vector fields on $X$ and $V_{0}(X) \subset V(X)$, the subset consisting of all vector fields with compact support. For $v \in V_{0}(X)$, for any $x \in X$, the curve

$$
t \mapsto \mathcal{V}_{t}^{v}(x) \in X
$$

is defined as the solution of the following Cauchy problem

$$
\begin{cases}\frac{d}{d t} \mathcal{V}_{t}^{v}(x) & =v\left(\mathcal{V}_{t}^{v}(x)\right), \\ \mathcal{V}_{0}^{v}(x) & =x\end{cases}
$$

The associated flow $\left(\mathcal{V}_{t}^{v}, t \in \mathbf{R}\right)$ induces a curve $\left(\mathcal{V}_{t}^{v}\right)^{*} \eta=\eta \circ\left(\mathcal{V}_{t}^{v}\right)^{-1}, t \in \mathbf{R}$, on $\Gamma_{X}$ : If $\eta=\sum_{x \in \eta} \varepsilon_{x}$ then $\left(\mathcal{V}_{t}^{v}\right)^{*} \eta=\sum_{x \in \eta} \varepsilon_{\mathcal{V}_{t}^{v}(x)}$.

Hypothesis I. Throughout this paper, we assume that $\mu$ (or $\nu$ ) is a Borel probability measure on $\Gamma_{X}$ such that the following conditions hold.

i) $\eta(\{x\}) \in\{0,1\}$ for all $x \in X$ and $\mu$-a.s. $\eta$.

ii) Either $\mu(\eta: \eta(X)<+\infty)=1$ or $\mu(\eta: \eta(X)=+\infty)=1$.

iii) For all $v \in V_{0}(X)$ and $t \in \mathbf{R}, \mu$ is quasi-invariant with respect to the flow $\left(\mathcal{V}_{t}^{v}\right)^{*}$ of $\Gamma_{X}$, i.e., $\mu \circ\left(\left(\mathcal{V}_{t}^{v}\right)^{*}\right)^{-1}$ is equivalent to $\mu$.

We are then in position to define the notion of differentiability on $\Gamma_{X}$. A measurable function $F: \Gamma_{X} \rightarrow \mathbf{R}$ is said to be differentiable if for any $v \in V_{0}(X)$, the following limit exists:

$$
\lim _{t \rightarrow 0} t^{-1}\left(F\left(\left(\mathcal{V}_{t}^{v}\right)^{*} \eta\right)-F(\eta)\right) .
$$

We then denote $\nabla_{v}^{\Gamma} F(\eta)$ the preceding limit and by $\nabla_{x}^{\Gamma} F(\eta)$ the corresponding gradient (see [AKR98]) which is defined by the identity:

$$
\int \nabla_{x}^{\Gamma} F(\eta) \cdot v(x) \mathrm{d} \eta(x)=\nabla_{v}^{\Gamma} F(\eta)
$$

for all $v \in V_{0}(X)$.

\section{WASSERSTEIN DISTANCE}

We consider on $X=\mathbf{R}^{k}$ the cost function as $d(x, y)=2^{-1}\|x-y\|^{2}$ where $\|x\|$ denote the euclidean norm of $x \in X$ and we define a cost between configurations (see also [BM02, BX00, Xia00]) as the 'lifting' of $d$ on $\Gamma_{X}$ :

$$
c\left(\eta_{1}, \eta_{2}\right)=\inf \left\{\int d(x, y) d \beta(x, y), \beta \in \Gamma_{\eta_{1}, \eta_{2}}\right\},
$$

where $\Gamma_{\eta_{1}, \eta_{2}}$ denotes the set of $\beta \in \Gamma_{X \times X}$ having marginals $\eta_{1}$ and $\eta_{2}$. According to [RS99], $c$ is lower semi continuous on $\Gamma_{X} \times \Gamma_{X}$. We can then set the Monge-Kantorovitch problem for configuration spaces.

Definition 1. Let $\mu$ and $\nu$ be two probability measures on $\left(\Gamma_{X}, \mathcal{B}\left(\Gamma_{X}\right)\right)$. We say that a probability $\gamma$ on $\left(\Gamma_{X} \times \Gamma_{X}, \mathcal{B}\left(\Gamma_{X} \times \Gamma_{X}\right)\right)$ is a solution of the Monge-Kantorovitch Problem associated to the couple $(\mu, \nu)$ and to the cost 
$c(M K P(\mu, \nu, c)$ for short) if the first marginal of $\gamma$ is $\mu$, the second one is $\nu$ and if

$$
\begin{aligned}
J(\gamma) & =\int c(\eta, \zeta) d \gamma(\eta, \zeta) \\
& =\inf \left\{\int c(\eta, \zeta) d \beta(\eta, \zeta): \beta \in \Sigma(\mu, \nu)\right\}
\end{aligned}
$$

Since $\Gamma_{X}$ is Polish, this infimum is attained and is equal to

$$
\sup \left\{\int F(\eta) \mathrm{d} \mu(\eta)+\int G(\zeta) \mathrm{d} \nu(\zeta):(F, G) \in \Phi_{c}\right\},
$$

where $\Phi_{c}$ is the set of pairs of measurable, real-valued functions $F$ and $G$ such that $F$ (resp. $G$ ) belongs to $L^{1}(\mathrm{~d} \mu)$ (resp. $\left.L^{1}(\mathrm{~d} \nu)\right)$ and $F(\eta)+G(\zeta) \leq$ $c(\eta, \zeta)$. The Wasserstein distance between $\mu$ and $\nu$ is the square root of $\mathcal{T}_{c}(\mu, \nu)$.

Since the cost $c$ is infinite whenever the two configurations do not have the same mass, we have the following theorem.

Theorem 3.1. Let $\mu$ and $\nu$ be two probability measures on the configuration space $\Gamma_{X}$. If the Monge-Kantorovitch cost, with respect to $c$, is finite then

$$
\mu(\eta(X)=n)=\nu(\eta(X)=n) \text { for any } n \in \mathbf{N} \cup\{+\infty\} .
$$

Proof. There exists at least one measure $\gamma$ such that

$$
\begin{aligned}
& \infty>\int c(\eta, \omega) \mathrm{d} \gamma(\eta, \omega) \\
&=\int_{\eta(X)=\omega(X)} c(\eta, \omega) \mathrm{d} \gamma(\eta, \omega)+\int_{\eta(X) \neq \omega(X)} c(\eta, \omega) \mathrm{d} \gamma(\eta, \omega) .
\end{aligned}
$$

This implies that $\gamma(\eta(X) \neq \omega(X))=0$. It follows that

$$
\begin{aligned}
\nu(\omega(X)=n) & =\gamma(\omega(X)=n) \\
& =\gamma(\omega(X)=n ; \eta(X)=n)+\gamma(\omega(X)=n ; \eta(X) \neq n) \\
& =\gamma(\omega(X)=n ; \eta(X)=n),
\end{aligned}
$$

for any $n \in \mathbf{N} \cup\{+\infty\}$. By the very same reasoning, it also holds that $\mu(\eta(X)=n)=\gamma(\omega(X)=n ; \eta(X)=n)$ for any $n$ and thus that $\mu(\eta(X)=$ $n)=\nu(\eta(X)=n)$ for any $n \in \mathbf{N} \cup\{+\infty\}$.

\section{Finite point PROCESSES}

Consider $\Lambda$ a compact set of $X$ and let $\zeta$ be fixed in $\Gamma_{\Lambda^{c}}$. We define $c_{\zeta}$ as:

$$
\begin{aligned}
& c_{\zeta}: \Gamma_{\Lambda} \times \Gamma_{X} \rightarrow \mathbf{R}^{+} \cup\{+\infty\} \\
& (\eta, \omega) \quad \mapsto c(\eta+\zeta, \omega) \text {. }
\end{aligned}
$$

Let $\mu$ be a probability measure on $\Gamma_{\Lambda}$ and $\nu$ a probability measure on $\Gamma_{X}$, we denote by $\mathcal{T}_{c_{\zeta}}(\mu, \nu)$ the $c_{\zeta}$-Wasserstein distance between $\mu$ and $\nu$ :

$$
\mathcal{T}_{c_{\zeta}}(\mu, \nu)=\inf _{\gamma \in \Sigma(\mu, \nu)} \int_{\Lambda \times X} c_{\zeta}(\eta, \omega) \mathrm{d} \gamma(\eta, \omega) .
$$

Since $\Lambda$ is compact and $E_{\mu} \eta$ is supposed to be a Radon measure, the configurations of $\Gamma_{\Lambda}$ have $\mu$-a.s. a finite number of atoms. It it then useful to 
think of $\Gamma_{\Lambda}$ as the disjoint union of the spaces $\Gamma_{\Lambda}^{(n)}$ for $n$ running from 0 to infinity, where

$$
\Gamma_{\Lambda}^{(n)}=\left\{\eta \in \Gamma_{\Lambda}, \eta(\Lambda)=n\right\} .
$$

Then, consider $\tilde{\Lambda}^{n}=\left\{\left(x_{1}, \cdots, x_{n}\right) \in \Lambda^{n}, x_{i} \neq x_{j}\right.$ for $\left.i \neq j\right\}$, there is a bijection $s_{\Lambda}^{n}$ between $\tilde{\Lambda}^{n} / n$ and $\Gamma_{\Lambda}^{(n)}$ :

$$
\begin{aligned}
& s_{\Lambda}^{n}: \quad \tilde{\Lambda}^{n} / n \quad \longrightarrow \Gamma_{\Lambda}^{(n)} \\
& \left\{x_{1}, \cdots, x_{n}\right\} \longmapsto \sum_{i=1}^{n} \varepsilon_{x_{i}} .
\end{aligned}
$$

The topology of $\tilde{\Lambda}^{n} / n$ induced by the usual topology of $\Lambda^{n}$ thus defines a locally compact metrizable Hausdorff topology on $\Gamma_{\Lambda}^{(n)}$. Since $\Lambda$ is compact, this topology coincides with the restriction to $\Gamma_{\Lambda}^{(n)}$ of the vague topology on $\Gamma_{\Lambda}$. We put on $\Gamma_{\Lambda}^{(n)}, \mathcal{B}\left(\Gamma_{\Lambda}^{(n)}\right)$ the associated Borel $\sigma$-algebra. For any map $F$ from $\Gamma_{\Lambda}$ into a measurable space $(Y, \mathcal{Y})$, for any integer $n$, we can consider, $F_{n}$, the restriction of $F_{n}$ to $\Gamma_{\Lambda}^{(n)}$ :

$$
\begin{aligned}
& F_{n}: \Gamma_{\Lambda}^{(n)} \rightarrow Y \\
& \eta \quad \mapsto \quad F_{n}(\eta)=F(\eta) .
\end{aligned}
$$

Since $\Gamma_{\Lambda}^{(n)}$ is closed in $\Gamma_{\Lambda}$, it is a Polish space and $F_{n}$ is measurable from $\left(\Gamma_{\Lambda}^{(n)}, \mathcal{B}\left(\Gamma_{\Lambda}^{(n)}\right)\right)$ into $(Y, \mathcal{Y})$.

We now identify $\sigma \in{ }_{n}$ and its action over $\tilde{\Lambda}^{n}$, which maps $x=\left(x_{1}, \cdots, x_{n}\right)$ to $\sigma x=\left(x_{\sigma(1)}, \cdots, x_{\sigma(n)}\right)$. Let $F_{n}^{s}$ be a measurable function from $\tilde{\Lambda}^{n}$ into a measurable space $(Y, \mathcal{Y})$. We say that $F_{n}^{s}$ is symmetric whenever for any $\sigma \in{ }_{n}, F^{s}(\sigma x)=F^{s}(x)$ for any $x \in \tilde{\Lambda}^{n}$. Identify now $\tilde{\Lambda}^{n} / n$ with a subset $\Lambda^{\prime}$ of $\tilde{\Lambda}^{n}$, since $\tilde{\Lambda}^{n}$ has $n$ ! disjoint connected components, the map

$$
\begin{aligned}
j^{n}: \tilde{\Lambda}^{n} & \longrightarrow \tilde{\Lambda}^{n} /{ }_{n} \times{ }_{n} \\
x & \longmapsto(\bar{x}, \sigma)=\left(j_{1}^{n}(x), j_{2}^{n}(x)\right),
\end{aligned}
$$

where $\sigma$ is such that $\sigma x=\bar{x} \in \Lambda^{\prime}$, is an homeomorphism. Furthermore, $j_{1}^{n}$ is a local diffeomorphism. Hence, any symmetric measurable (respectively continuous or differentiable) function $F_{n}^{s}$ from $\tilde{\Lambda}^{n}$ into $\mathbf{R}$ can be identified with a measurable (respectively continuous or differentiable) function $F_{n}$ from $\Gamma_{\Lambda}^{(n)}$ into $\mathbf{R}$ with

$$
F_{n}(\eta)=F_{n}^{s}\left(j^{-1}\left(\left(s_{\Lambda}^{n}\right)^{-1}(\eta), \sigma\right)\right)
$$

for any $\sigma \in{ }_{n}$ or equivalently with

$$
F_{n}(\eta)=\frac{1}{n !} \sum_{\sigma \in \in_{n}} F_{n}^{s}\left(j^{-1}\left(\left(s_{\Lambda}^{n}\right)^{-1}(\eta), \sigma\right)\right) .
$$

Conversely, any function $F_{n}$ from $\Gamma_{\Lambda}^{(n)}$ into $\mathbf{R}$ gives raise to a symmetric function $F_{n}^{s}$ from $\tilde{\Lambda}^{n}$ into $\mathbf{R}$ by $F_{n}^{s}(x)=F\left(s_{\Lambda}^{n}\left(j_{1}(x)\right)\right)$, with the same regularity (measurable, continuous or differentiable). Accordingly, every probability measure $\mu_{n}$ on $\Gamma_{\Lambda}^{(n)}$ can be viewed as a symmetric (i.e., invariant under the action of ${ }_{n}$ ) probability measure $\mu_{n}^{s}$ on $\tilde{\Lambda}^{n}$ and vice-versa. 
Let $\mu$ be a probability measure on $\Gamma_{\Lambda}$ and consider the disintegration of $\mu$ along the map $(\eta \mapsto \eta(\Lambda))$ :

$$
\mu(B)=\sum_{n \geq 0} \mu(B \mid \eta(\Lambda)=n) \mathrm{P}(\eta(\Lambda)=n) .
$$

We denote by $\mu_{n}$ the measure $\mu(. \mid \eta(\Lambda)=n)$. The measure $\mathrm{P}(\eta(\Lambda)=n) \mu_{n}$ is the so-called Janossy measure of order $n$ (see [DVJ03]). We say that $\mu$ is regular whenever for any $n \geq 1, \mu_{n}^{s}$, the symmetric measure associated to $\mu_{n}$, is absolutely continuous with respect to the Lebesgue measure on $\tilde{\Lambda}^{n}$.

Remark 4.1. Since $X$ is Polish it can embedded as a $G_{\delta}$ in a compact metric space $X^{\prime}$. If a probability measure $\nu$ on $\Gamma_{X}$ is such that $\nu(\omega(X)<+\infty)=$ 1 , we can embed $\left(\Gamma_{X}, \mathcal{B}\left(\Gamma_{X}\right), \nu\right)$ into $\left(\Gamma_{X^{\prime}}, \mathcal{B}\left(\Gamma_{X^{\prime}}\right), \nu_{X^{\prime}}\right)$, with $\operatorname{supp} \nu_{X^{\prime}}=$ $\operatorname{supp} \nu$ and $\nu_{X^{\prime}}(\omega(X)<+\infty)=1$. Thus, all the previous results established on $\Gamma_{\Lambda}$ are valid on $\Gamma_{X^{\prime}}$ hence on $\Gamma_{X}$. In particular to every probability measure $\nu_{n}$ on $\Gamma_{X} \subset \Gamma_{X^{\prime}}$, we can associate, as above, a symmetric probability measure, $\nu_{n}^{s}$ on $\tilde{X}^{n}$.

The next theorem follows from the previous considerations.

Theorem 4.1. Assume that $\mu$ is a regular probability measure on $\Gamma_{\Lambda}$ and let $F$ be measurable from $\Gamma_{\Lambda}$ into $\mathbf{R}$. Then, $F$ is $\mu$-a.s. differentiable, on its domain, if and only if $F_{n}^{s}$ is $\mu_{n}^{s}$-a.s. differentiable, on its domain, for any integer $n$.

The euclidean symmetric cost on $X^{n}$, denoted by $c_{n}^{s}$, is defined as:

$$
c_{n}^{s}(x, y)=\inf _{\sigma \in_{n}} \frac{1}{2}\|x-\sigma y\|^{2} .
$$

It is immediate that

$$
c_{n}^{s}(x, y)=c\left(s_{\Lambda}^{n}\left(j_{1}^{n}(x)\right), s_{\Lambda}^{n}\left(j_{1}^{n}(y)\right)\right)
$$

and that

$$
c(\eta, \omega)=c_{n}^{s}(x, y)
$$

for any $x \in\left(s_{\Lambda}^{n} \circ j_{1}^{n}\right)^{-1}(\{\eta\})$ and any $y \in\left(s_{\Lambda}^{n} \circ j_{1}^{n}\right)^{-1}(\{\omega\})$.

Lemma 4.1. A function $F_{n}^{s}$ from $\tilde{\Lambda}^{n}$ into $\mathbf{R}$ is $c_{n}^{s}$-concave if and only if $F_{n}^{s}-\|x\|^{2} / 2$ is concave in the usual sense and $F_{n}^{s}$ is symmetric.

Proof. By its very definition, a $c_{n}^{s}$-concave function $F_{n}^{s}$ is of the form:

$$
F_{n}^{s}(x)=\inf _{i \in I}\left(c_{n}^{s}\left(x, y_{i}\right)+a_{i}\right)=\inf _{\substack{i \in I \\ \sigma \in n}}\left(\frac{1}{2}\left\|x-\sigma y_{i}\right\|^{2}+a_{i}\right),
$$

where $y_{i}$ belongs to $\tilde{\Lambda}^{n}$ for any $i \in I$. This clearly implies that $F_{n}^{s}$ is symmetric and euclidean-concave and euclidean-concavity is known to be equivalent to the concavity of $\left(x \mapsto F_{n}^{s}(x)-\|x\|^{2} / 2\right)$ in the usual sense (see [Vil03]), hence the result.

It only remains to prove that $F_{n}^{s}$ symmetric and euclidean-concave can be written as in (5). Since $F_{n}^{s}$ is euclidean concave,

$$
F_{n}^{s}(x)=\inf _{i \in I}\left(\frac{1}{2}\left\|x-y_{i}\right\|^{2}+a_{i}\right),
$$


for some index set $I,\left(a_{i}, i \in I\right)$ a family of real numbers and $\left(y_{i}, i \in I\right)$ some elements of $X^{n}$. Since $F_{n}^{s}$ is symmetric, $F_{n}^{s}(x)=F_{n}^{s}(\sigma x)=\inf _{\sigma \in{ }_{n}} F_{n}^{s}(\sigma(x))$ thus

$$
F_{n}^{s}(x)=\inf _{\substack{i \in I \\ \sigma \in n}}\left(\frac{1}{2}\left\|\sigma x-y_{i}\right\|^{2}+a_{i}\right)=\inf _{\substack{i \in I \\ \sigma \in n}}\left(\frac{1}{2}\left\|x-\sigma y_{i}\right\|^{2}+a_{i}\right) .
$$

The proof is thus complete.

It follows from the Lebesgue-a.s. differentiability of concave function that we have:

Corollary 4.1. Let $n \geq 1$ and $F_{n}^{s}$ be a $c_{n}^{s}$-concave function. Then, $F_{n}^{s}$ is Lebesgue-a.s. differentiable on its domain.

Corollary 4.2. Let $n \geq 1, \mu_{n}^{s}$ an absolutely continuous measure on $\tilde{\Lambda}^{n}$ and $F_{n}^{s}$ a $c_{n}^{s}$-concave function. Then, $\partial_{c_{n}^{s}} F^{s}$ is $\mu_{n}^{s}$-a.s. single-valued.

Proof. We already know (see Corollary 4.1) that $F_{n}^{s}$ is Lebesgue-a.s. differentiable. From (4), it is clear that

$$
\partial^{c_{n}^{s}} F_{n}^{s}(x)=\partial^{c_{\emptyset}} F_{n}\left(s_{\Lambda}^{n}\left(j_{1}^{n}(x)\right)\right) .
$$

The previous theorem implies that the rightmost set is reduced to a singleton for $\mu_{n}^{s}$-almost-all $x$, hence the result.

Remind now that for two configurations $\eta+\zeta$ and $\omega$ at finite $c$ distance, $\beta_{\eta+\zeta, \omega}$ is one measure on $\Gamma_{X \times X}$ which realizes this distance.

Definition 2. For any $\eta \in \Gamma_{X}$, for any $\Lambda \subset X, \pi^{\Lambda}(\eta)=\eta \cap \Lambda$. For any map $t$ from $X$ to $X$, we associate the map $t^{\Gamma}$ from $\Gamma_{X}$ to itself, defined by

$$
t^{\Gamma}\left(\sum_{x \in \eta} \varepsilon_{x}\right)=\sum_{x \in \eta} \varepsilon_{t(x)} \text { for any } \eta=\sum_{x \in \eta} \varepsilon_{x}
$$

For any probability measure $\mu$ on $\Gamma_{X}, \pi^{\Lambda} \mu$ is the image measure of $\mu$ by $\pi^{\Lambda}$. For any $\eta=\left(\eta_{1}, \eta_{2}\right) \in \Gamma_{X} \times \Gamma_{X}$, we set $p_{i}(\eta)=\eta_{i}$ for $i=1$, 2. Accordingly, for any probability measure $\gamma$ on $\Gamma_{X} \times \Gamma_{X}, p_{i} \gamma$ the image of $\gamma$ by $p_{i}$. We also introduce $\pi_{i}^{\Lambda}:=\pi^{\Lambda} \circ p_{i}$, thus $\pi_{1}^{\Lambda}(\eta, \omega)$ is the restriction to $\Lambda$ of $\eta$. For any configuration $\beta$ on $X \times X$, define $r^{\Lambda}$ by:

$$
\begin{aligned}
r^{\Lambda}: \Gamma_{X \times X} & \rightarrow \Gamma_{X \times X} \\
\beta & \mapsto r^{\Lambda} \beta=\beta \cap(\Lambda \times X) .
\end{aligned}
$$

At last, $r_{i}^{\Lambda}$ denotes $p_{i} \circ r^{\Lambda}$.

The main result of this section is the following.

Theorem 4.2. Let $\mu$ (resp. $\nu$ ) be a probability measure on $\Gamma_{\Lambda}$ (resp. $\Gamma_{X}$ ) satisfying Hypothesis $\square$ and $\zeta \in \Gamma_{\Lambda^{c}}$. Assume that $\mu$ is regular and that $\mathcal{T}_{c_{\zeta}}(\mu, \nu)$ is finite. Then, for any optimal measure $\rho$, there exists a $c_{\zeta}$-concave function $F$ such that $\operatorname{supp} \rho \subset \partial^{c_{\zeta}} F$ and for any $\omega \in \partial^{c_{\zeta}} F(\eta)$,

$$
r^{\Lambda}\left(\beta_{\eta+\zeta, \omega}\right)=\sum_{x \in \eta} \varepsilon_{\left(x, x-\nabla_{x}^{\Gamma} F(\eta)\right)},
$$

for any $\beta_{\eta+\zeta, \omega}$ realizing $c(\eta+\zeta, \omega)$. 
Proof. $\Gamma_{\Lambda}$ and $\Gamma_{X}$ are Polish spaces hence there exists at least an optimal measure $\rho$ and a $c_{\zeta}$-concave function $F$ such that supp $\rho \subset \partial^{c} \zeta F$. By the definition of $c_{\zeta}$-concavity, for any $\eta \in \Gamma_{\Lambda}$,

$$
\begin{aligned}
F(\eta) & =\inf _{i \in I}\left(c\left(\eta+\zeta, \omega_{i}\right)+a_{i}\right) \\
& =\inf _{i \in I} \inf _{\varpi_{i} \subset \omega_{i}}\left(c\left(\eta, \varpi_{i}\right)+c\left(\zeta, \varpi_{i}^{c}\right)+a_{i}\right)
\end{aligned}
$$

Since $c\left(\zeta, \varpi_{i}^{c}\right)+a_{i}$ does not depend on $\eta, F_{n}^{s}$ is $c_{n}^{s}$-concave for any integer $n$. Then Corollary 4.1 implies that $F_{n}^{s}$ is Lebesgue-a.s. differentiable, which in turn entails that $F_{n}^{s}$ is $\mu_{n}^{s}$-a.s. differentiable, since $\mu_{n}^{s}$ is absolutely continuous with respect to the Lebesgue measure. Thus, according to Corollary 4.1 and Theorem 4.1. $F$ has $\mu$-a.s. directional derivatives for any $v \in V_{0}(\Lambda)$. Let $v \in V_{0}(\Lambda)$, any $\omega \in \partial^{c_{\zeta}} F(\eta)$ must satisfy

$$
F\left(\left(\mathcal{V}_{t}^{v}\right)^{*} \eta\right)-F(\eta) \leq c\left(\left(\mathcal{V}_{t}^{v}\right)^{*} \eta+\zeta, \omega\right)-c(\eta+\zeta, \omega),
$$

for any $t \in \mathbf{R}$ and $c(\eta+\zeta, \omega)<+\infty$. For any $\beta_{\eta+\zeta, \omega}$ realizing $c(\eta+\zeta, \omega)$,

$$
\begin{aligned}
c\left(\left(\mathcal{V}_{t}^{v}\right)^{*} \eta+\zeta, \omega\right) \leq \frac{1}{2} \int_{\Lambda \times X}\left\|\mathcal{V}_{t}^{v}(x)-y\right\|^{2} \mathrm{~d} & \beta_{\eta+\zeta, \omega} \\
& +\frac{1}{2} \int_{\Lambda^{c} \times X}\|x-y\|^{2} \mathrm{~d} \beta_{\eta+\zeta, \omega} .
\end{aligned}
$$

Hence,

$$
F\left(\left(\mathcal{V}_{t}^{v}\right)^{*} \eta\right)-F(\eta) \leq \frac{1}{2} \int_{\Lambda \times X}\left(\left\|\mathcal{V}_{t}^{v}(x)-y\right\|^{2}-\|x-y\|^{2}\right) \mathrm{d} \beta_{\eta+\zeta, \omega} .
$$

Divide the two terms of this inequality by $t>0$ and let $t$ go to 0 , we get

$$
\frac{d}{d t} F\left(\left(\mathcal{V}_{t}^{v}\right)^{*} \eta\right)_{\mid t=0} \leq \int_{\Lambda \times X}(x-y) \cdot v(x) \mathrm{d} \beta_{\eta+\zeta, \omega}(x, y) .
$$

Applying this inequality to $-v$, we deduce that for any $v \in V_{0}(\Lambda)$,

$$
\nabla_{v}^{\Gamma} F(\eta)=\int_{\Lambda \times X}(x-y) \cdot v(x) \mathrm{d} \beta_{\eta+\zeta, \omega}(x, y) .
$$

We infer from this relation that for any $\omega \in \partial^{c} \zeta F(\eta)$,

$$
r^{\Lambda}\left(\beta_{\eta+\zeta, \omega}\right)=\sum_{x \in \eta} \varepsilon_{\left(x, \operatorname{Id}-\nabla_{x}^{\Gamma} F(\eta)\right)},
$$

for any $\beta_{\eta+\zeta, \omega}$ realizing $c(\eta+\zeta, \omega)$.

The last theorem means that only a part of any element $\omega$ of $\partial^{c_{\zeta}} F(\eta)$ is uniquely determined, namely the part which will be married to $\eta$ in an optimal coupling between $\omega$ and $\eta+\zeta$. Nonetheless, when $\zeta=\emptyset$, this means that $\partial^{c_{\emptyset}}(\eta)$ is reduced to one point which is $\left(\operatorname{Id}-\nabla^{\Gamma} F\right)^{\Gamma}(\eta)=\sum_{x \in \eta} \varepsilon_{x-\nabla_{x}^{\Gamma} F(\eta)}$.

Corollary 4.3. Assume that $\mu_{n}^{s}$ and $\nu_{n}^{s}$ are two absolutely continuous, symmetric, probability measures on $\tilde{\Lambda}^{n}$ and that $\mathcal{T}_{c_{n}^{s}}\left(\mu_{n}^{s}, \nu_{n}^{s}\right)$ is finite. Then there exists a unique optimal measure $\rho_{n}$ for $\operatorname{MKP}\left(\mu_{n}^{s}, \nu_{n}^{s}, c_{n}^{s}\right)$ and there exists a unique map $t_{n}^{s}$ such that $\rho_{n}=\left(\operatorname{Id} \otimes t_{n}^{s}\right)^{*} \mu_{n}^{s}$. 
Proof. View $\tilde{\Lambda}^{n}$ as a subset of the Polish space $\Lambda^{n}$. Since $\Lambda^{n} \backslash \tilde{\Lambda}^{n}$ has a null Lebesgue measure, we can then view $\mu_{n}^{s}$ and $\nu_{n}^{s}$ as absolutely continuous, symmetric, probability measures on $\Lambda^{n}$. Since $\Lambda^{n}$ is Polish, there exists at least one optimal measure for $\operatorname{MKP}\left(\mu_{n}^{s}, \nu_{n}^{s}, c_{n}^{s}\right)$. For any optimal measure $\rho$, there exists a $c_{n}^{s}$-concave function $f_{n}$ such that $\operatorname{supp} \rho \subset \partial_{c_{n}^{s}} f_{n}$. According to Corollary 4.2, $\partial_{c_{n}^{s}} f_{n}$ is $\mu_{n}^{s}$-a.s. single-valued, hence the uniqueness of $\rho_{n}$ and $t_{n}$ follows from Theorem 2.1

We can then state:

Theorem 4.3. Let $\mu$ be a regular probability measure on $\Gamma_{\Lambda}$ and $\nu$ be a probability measure on $\Gamma_{X}$. The Monge-Kantorovitch distance, associated to $c$, between $\mu$ and $\nu$ is finite if and only if the following two conditions hold

(a) $\mu(\eta(\Lambda)=n)=\nu(\omega(X)=n)$ for any integer $n \geq 0$,

(b) $\sum_{n \geq 1} \mathcal{T}_{c}\left(\mu_{n}, \nu_{n}\right)^{2} \mu(\eta(\Lambda)=n)$ is finite.

Moreover, we have

$$
\mathcal{T}_{c}(\mu, \nu)^{2}=\sum_{n \geq 1} \mathcal{T}_{c}\left(\mu_{n}, \nu_{n}\right)^{2} \mu(\eta(\Lambda)=n),
$$

and there exists a unique c-concave map $F$ such that the unique optimal measure $\rho$ is given by

$$
\rho=\left(\operatorname{Id} \otimes\left(\operatorname{Id}-\nabla^{\Gamma} F\right)^{\Gamma}\right)^{*} \mu,
$$

where

$$
\left(\mathrm{Id}-\nabla^{\Gamma} F\right)^{\Gamma}(\eta)=\sum_{x \in \eta} \varepsilon_{x-\nabla_{x}^{\Gamma} F(\eta)}
$$

Proof. If $\mathcal{T}_{c}(\mu, \nu)$ is finite then according to Theorem 3.1 condition (ai) is satisfied. Thus, we have

$$
\begin{aligned}
\mathcal{T}_{c}(\mu, \nu)^{2} & =\inf _{\gamma \in \Sigma(\mu, \nu)} \sum_{n \geq 1} \int_{\eta(\Lambda)=\omega(X)=n} c(\eta, \omega) \mathrm{d} \gamma(\eta, \omega) \\
& =\inf _{\gamma \in \Sigma(\mu, \nu)} \sum_{n \geq 1} \int_{\Gamma_{\Lambda}^{n} \times \Gamma_{X}} c(\eta, \omega) \mathrm{d}(\gamma \mid \eta(\Lambda)=n)(\eta, \omega) \mu(\eta(\Lambda)=n) \\
& =\sum_{n \geq 1} \inf _{\gamma_{n} \in \Sigma\left(\mu_{n}, \nu_{n}\right)} \int_{\Gamma_{\Lambda}^{n} \times \Gamma_{X}} c(\eta, \omega) \mathrm{d} \gamma_{n}(\eta, \omega) \mu(\eta(\Lambda)=n) \\
& =\sum_{n \geq 1} \mathcal{T}_{c_{n}^{s}}\left(\mu_{n}^{s}, \nu_{n}^{s}\right)^{2} \mu(\eta(\Lambda)=n) \\
& =\sum_{n \geq 1} \mathcal{T}_{c}\left(\mu_{n}, \nu_{n}\right)^{2} \mu(\eta(\Lambda)=n),
\end{aligned}
$$

where $\mu_{n}^{s}$ (resp. $\nu_{n}^{s}$ ) is the symmetric measure on $\tilde{\Lambda}^{n}$ corresponding to $\mu_{n}$ (resp. $\mu_{n}^{s}$ ). Let $\rho$ an optimal measure whose existence is guaranteed because $\Gamma_{\Lambda}$ and $\Gamma_{X}$ are Polish, we infer from Theorem 4.2 that there exists a $c^{\emptyset}$ concave function $F$ whose $c^{\emptyset}$-super-gradient is $\mu$-a.s. single valued such that $\operatorname{supp} \rho \subset \partial^{c \emptyset} F$. According to Theorem 2.1] this implies that $\rho$ and $T$ are unique and that $\rho=(\operatorname{Id} \otimes T)^{*} \mu$. At last, Theorem 4.2 entails that $T=$ $\left(\mathrm{Id}-\nabla^{\Gamma} F\right)^{\Gamma}$. 
In the converse direction, since $\mu$ is regular and $\mathcal{T}_{c}\left(\mu_{n}, \nu_{n}\right)=\mathcal{T}_{c_{n}^{s}}\left(\mu_{n}^{s}, \nu_{n}^{s}\right)$ is finite for any $n \geq 1$, there exists, for any $n \geq 1$, according to Corollary 4.3. a measure $\rho_{n}^{s}$ such that

$$
\mathcal{T}_{c_{n}^{s}}\left(\mu_{n}^{s}, \nu_{n}^{s}\right)^{2}=\int_{\Lambda \times X} c_{n}^{s}(x, y) \mathrm{d} \rho_{n}(x, y) .
$$

Now, we set

$$
\rho(A)=\sum_{n \geq 1} \rho_{n}\left(A \cap\left(\Gamma_{\Lambda}^{(n)} \times \Gamma_{X}\right)\right) \mu(\eta(\Lambda)=n) .
$$

Since $\mu(\eta(\Lambda)=n)=\nu(\eta(\Lambda)=n)$ and since $\rho_{n}^{s}$ belongs to $\Sigma\left(\mu_{n}, \nu_{n}\right)$, it is clear that $\rho$ belongs to $\Sigma(\mu, \nu)$. Moreover, we have:

$$
\begin{aligned}
\int_{\Gamma_{\Lambda} \times \Gamma_{X}} c(\eta, \omega) \mathrm{d} \rho(\eta, \omega) & =\sum_{n \geq 1} \mu(\eta(\Lambda)=n) \int_{\Gamma_{\Lambda}^{(n)} \times \Gamma_{X}} c(\eta, \omega) \mathrm{d} \rho_{n}(\eta, \omega) \\
& =\sum_{n \geq 1} \mu(\eta(\Lambda)=n) \int_{\Lambda^{n} \times X^{n}} c_{n}^{s}(x, y) \mathrm{d} \rho_{n}^{s}(x, y) \\
& =\sum_{n \geq 1} \mu(\eta(\Lambda)=n) \mathcal{T}_{c_{n}^{s}}\left(\mu_{n}^{s}, \nu_{n}^{s}\right)^{2},
\end{aligned}
$$

and the last quantity is finite according to the hypothesis. Thus, $\tau_{c}(\mu, \nu)$ is finite. It remains to prove that $\rho$ constructed above is optimal. For, remind that, as mentioned in the preliminaries, it is sufficient that $\operatorname{supp} \rho$ be $c$ cyclically monotone. We infer from the finiteness of $\int c \mathrm{~d} \rho$ that for any $(\eta, \omega)$ in $\operatorname{supp} \rho, \eta(\Lambda)=\omega(X)$. For $m$ any integer, let $\left(\left(\eta_{i}, \omega_{i}\right), i=1, \cdots, m\right)$ be a family of elements of $\operatorname{supp} \rho$. Set $I_{n}=\left\{i \in 1, \cdots, m, \eta_{i}(\Lambda)=n\right\}$, we can then write

$$
\sum_{i=1}^{m} c\left(\eta_{i}, \omega_{i}\right)=\sum_{n=1}^{+\infty} \sum_{i \in I_{n}} c\left(\eta_{i}, \omega_{i}\right) .
$$

Let $\sigma \in{ }_{m}$, if for some $n, \sigma I_{n}$ differs from $I_{n}$ then $\sum_{i \in I_{n}} c\left(\eta_{i}, \omega_{i}\right)$ is infinite and it is clear that

$$
\sum_{i=1}^{m} c\left(\eta_{i}, \omega_{i}\right) \leq \sum_{i=1}^{m} c\left(\eta_{i}, \omega_{\sigma(i)}\right) .
$$

Thus, we now assume that for any $n \geq 1, \sigma I_{n}=I_{n}$, i.e., $\omega_{i}(\Lambda)=\omega_{\sigma(i)}(\Lambda)$ for any $i=1, \cdots, m$. Since for any $n \geq 1, \rho_{n}^{s}$ is $c_{n}^{s}$-cyclically monotone, so does $\rho_{n}$. Moreover, $\operatorname{supp} \rho \cap I_{n}=\rho_{n}$, thus for any $n \geq 1$,

$$
\sum_{i \in I_{n}} c\left(\eta_{i}, \omega_{i}\right) \leq \sum_{i \in I_{n}} c\left(\eta_{i}, \omega_{\sigma(i)}\right) \text {. }
$$

By summation, we infer that $\sum_{i=1}^{m} c\left(\eta_{i}, \omega_{i}\right) \leq \sum_{i=1}^{m} c\left(\eta_{i}, \omega_{\sigma(i)}\right)$ for any $\sigma \in$ $m$. This amounts to say that $\operatorname{supp} \rho$ is $c$-cyclically monotone, hence that $\rho$ is an optimal measure (unique according to the first part of the proof) for $\operatorname{MKP}(\mu, \nu, c)$. We deduce from (7) that (6) holds true. 
4.1. Example : Wasserstein distance with respect to a Poisson process. Let $\sigma$ be a diffuse (by which we mean absolutely continuous with respect to the Lebesgue measure) Radon measure on $X$, the Poisson measure on $\Gamma_{X}$ with intensity $\sigma$, denoted by $\mu_{\sigma}$, is the unique probability measure on $\left(\Gamma_{X}, \mathcal{B}\left(\Gamma_{X}\right)\right)$ such that

$$
E\left[\exp \left(\int f \mathrm{~d} \eta\right)\right]=\exp \left(\int_{X}\left(e^{f(x)}-1\right) \mathrm{d} \sigma(x)\right),
$$

for all $f \in \mathcal{C}_{0}$. It is well known that $\mu_{\sigma}$ satisfies Hypothesis \and $\mu_{\sigma}$ is regular since $\mu_{n}=\sigma^{\otimes n}$, thus the previous results apply. Let $\sigma_{1}$ and $\sigma_{2}$ two diffuse probability measures on $X$ with finite Wasserstein distance with respect to the euclidean cost on $X=\mathbf{R}^{k}$ :

$$
\mathcal{T}_{e}\left(\sigma_{1}, \sigma_{2}\right)^{2}=\inf _{\gamma \in \Sigma\left(\sigma_{1}, \sigma_{2}\right)} \frac{1}{2} \int_{X \times X}\|x-y\|^{2} \mathrm{~d} \gamma(x, y)<+\infty .
$$

We denote by $t$ the transport map from $\sigma_{1}$ to $\sigma_{2}$ and $\phi$ its potential, i.e., the convex map from $X \rightarrow \mathbf{R}$ such that $\nabla \phi=t$. By $\nabla$, we mean here the usual gradient in $X$. For $W$ and $Y$ two spaces and $f: W \rightarrow \mathbf{R}$ and $g: Y \rightarrow \mathbf{R}$, we denote by $f \oplus g$ the map defined on $W \times Y$ by $(f \oplus g)(x, y)=f(x)+g(y)$.

Lemma 4.2. The map $t^{\otimes(n)}:\left(x_{1}, \ldots, x_{n}\right) \in X^{n} \mapsto\left(t\left(x_{1}\right), \ldots, t\left(x_{n}\right)\right)$ is the transport map from $\sigma_{1}^{\otimes n}$ to $\sigma_{2}^{\otimes n}$. Moreover,

$$
\mathcal{T}_{e}\left(\sigma_{1}^{\otimes n}, \sigma_{2}^{\otimes n}\right)^{2}=n \mathcal{T}_{e}\left(\sigma_{1}, \sigma_{2}\right)^{2} .
$$

Proof. It is immediate that $t^{\otimes(n)} \equiv \nabla\left(\oplus_{i=1}^{n} \phi\right)$ and that $\oplus_{i=1}^{n} \phi$ is convex, thus $t^{\otimes(n)}$ is cyclically monotone (with respect to the squared euclidean cost on $\left.X^{n}\right)$. Moreover, $\left(t^{\otimes(n)}\right)^{*}\left(\nu_{1}^{\otimes n}\right)=\nu_{2}^{\otimes n}$, hence $t^{\otimes(n)}$ is the optimal transport map between $\nu_{1}^{\otimes n}$ and $\nu_{2}^{\otimes n}$. Then,

$$
\begin{aligned}
\mathcal{T}_{e}\left(\sigma_{1}^{\otimes n}, \sigma_{2}^{\otimes n}\right)^{2} & =\int_{X^{n}} \frac{1}{2}\left\|x-t^{\otimes(n)}(x)\right\|^{2} \mathrm{~d} \sigma_{1}^{\otimes n}(x) \\
& =\sum_{j=1}^{n} \int_{X} \frac{1}{2}\left\|x_{j}-t^{\otimes(n)}\left(x_{j}\right)\right\|^{2} \mathrm{~d} \sigma_{1}\left(x_{j}\right) \\
& =n \mathcal{T}_{e}\left(\sigma_{1}, \sigma_{2}\right)^{2} .
\end{aligned}
$$

The proof is thus complete.

It then follows from Theorem 4.3 that:

Theorem 4.4. For $\sigma_{1}$ and $\sigma_{2}$ two diffuse probability measures on $X$, if $\mathcal{T}_{e}\left(\sigma_{1}, \sigma_{2}\right)<+\infty$ then $\mathcal{T}_{c}\left(\mu_{\sigma_{1}}, \mu_{\sigma_{2}}\right)$ is finite. If $t=\nabla \phi$ are respectively the transport map and its associated potential for $\operatorname{MKP}\left(\sigma_{1}, \sigma_{2}, c_{e}\right)$ then

$$
T=\sum_{n \geq 1} t^{\otimes(n)} \mathbf{1}_{\Gamma_{X}^{(n)}} \text { and } \Phi=\sum_{n \geq 1}\left(\oplus_{i=1}^{n} \phi\right)^{\Gamma} \mathbf{1}_{\Gamma_{X}^{(n)}},
$$

are respectively the transport map and the associated potential for the MongeKantorovitch problem $\operatorname{MKP}\left(\mu_{\sigma_{1}}, \mu_{\sigma_{2}}, c\right)$. Moreover,

$$
\mathcal{T}_{c}\left(\mu_{\sigma_{1}}, \mu_{\sigma_{2}}\right)=\mathcal{T}_{e}\left(\sigma_{1}, \sigma_{2}\right) \text {. }
$$


Remark 4.2. For finite point processes, it is possible to define a cost between configurations by

$$
c_{b}(\eta, \omega)=\frac{1}{\eta(X)} c(\eta, \omega)
$$

We would then have

$$
\mathcal{T}_{c_{b}}\left(\mu_{\sigma_{1}}, \mu_{\sigma_{2}}\right)^{2}=\left(1-e^{-1}\right) \mathcal{T}_{e}\left(\sigma_{1}, \sigma_{2}\right)^{2} .
$$

This distance $\mathcal{T}_{c_{b}}$ appears in papers of Barbour et al. [BB92, Xia00].

A Cox process is a doubly-stochastic Poisson process: $\sigma$ is now a random variable in the set of diffuse Radon measures on $X$ and conditionally to $\sigma$, the point process is a Poisson process of intensity $\sigma$. By conditioning with respect to the intensities, the proof given above yields to the following theorem.

Theorem 4.5. If $\mu$ and $\nu$ are two Cox processes of random intensities $\sigma_{1}$ and $\sigma_{2}$ respectively, such that $\mathbf{E}\left[\mathcal{T}_{e}\left(\sigma_{1}, \sigma_{2}\right)\right]$ is finite. Then,

$$
\mathcal{T}_{c}\left(\mu_{\sigma_{1}}, \mu_{\sigma_{2}}\right)=\mathbf{E}\left[\mathcal{T}_{e}\left(\sigma_{1}, \sigma_{2}\right)\right]
$$

\section{LOCALLY FINITE POINT PROCESSES}

We now only assume that $\mu$ is the law of a locally finite point process : $\mu(\eta(\Lambda)<+\infty)=1$ for all compact sets $\Lambda$ but $\mu(\eta(X)=+\infty)=1$. We can no longer work on the graded space $\cup_{n \geq 1} \Gamma_{X}^{(n)}$ since it is $\mu$-negligible. We are in fact reminded the case of the Wiener space. There is thus no big surprise that we can follow closely the beautiful method of [FÜ04].

Lemma 5.1. Let $\mu$ and $\nu$ be two probability measures on $\Gamma_{X}$ such that $\mathcal{T}_{c}(\mu, \nu)$ is finite. Let $\gamma$ be one optimal measure and $\Lambda$ be a compact set of $X$. Consider the disintegration of $\gamma$ along the projection $\pi_{1}^{\Lambda^{c}}$, i.e.,

$$
\gamma(.)=\int_{\Gamma_{\Lambda^{c}}} \gamma\left(. \mid \pi_{1}^{\Lambda^{c}}(\eta, \omega)=\eta_{\Lambda^{c}}\right) d \mu_{\Lambda^{c}}\left(\eta_{\Lambda^{c}}\right),
$$

where $\mu_{\Lambda^{c}}$ is the image measure of $\mu$ by $\pi^{\Lambda^{c}}$. Denote by $\gamma\left(. \mid \eta_{\Lambda^{c}}\right)$ the regular version of the conditional probability $\gamma\left(. \mid \pi_{1}^{\Lambda^{c}}(\eta, \omega)=\eta_{\Lambda^{c}}\right)$. Then, $\mu_{\Lambda^{c}}$-a.s., $\gamma\left(. \mid \eta_{\Lambda^{c}}\right)$ is an optimal measure for $\operatorname{MKP}\left(p_{1} \gamma\left(. \mid \eta_{\Lambda^{c}}\right), p_{2} \gamma\left(. \mid \eta_{\Lambda^{c}}\right), c_{\eta_{\Lambda^{c}}}\right)$.

Remark 5.1. If we denote by $(N, M)$ a couple of random variables whose distribution is $\gamma$ and if $N_{\Lambda}(\eta, \omega):=N(\eta) \cap \Lambda$, then the previous lemma stands that conditionally to $\left(N_{\Lambda^{c}}=\eta_{\Lambda^{c}}\right)$, the law of $\left(\eta_{\Lambda^{c}}+N_{\Lambda}, M\right)$ is optimal for $\operatorname{MKP}\left(\gamma_{\eta_{\Lambda^{c}}+N_{\Lambda} \mid N_{\Lambda^{c}}=\eta_{\Lambda^{c}}}, \gamma_{M \mid N_{\Lambda^{c}}=\eta_{\Lambda^{c}}}, c_{\eta_{\Lambda}^{c}}\right)$. Note that within this setting, since the law of $N$ is $\mu$, it is clear that

$$
\gamma_{N \mid N_{\Lambda^{c}}=\eta_{\Lambda^{c}}}=\mu_{N \mid N_{\Lambda^{c}}=\eta_{\Lambda^{c}}} \text { i.e., } p_{1} \gamma\left(. \mid \eta_{\Lambda^{c}}\right)=\mu\left(. \mid \eta_{\Lambda^{c}}\right) \text {. }
$$


Proof of Lemma 5.1. According to the definition of an optimal measure,

$$
\begin{aligned}
J_{c}(\gamma) & =\int c(\eta, \omega) \mathrm{d} \gamma(\eta, \omega) \\
& =\int c\left(\pi^{\Lambda} \eta+\pi^{\Lambda^{c}} \eta, \omega\right) \mathrm{d} \gamma(\eta, \omega) \\
& =\int_{\Gamma_{\Lambda^{c}}} \mathrm{~d} \mu_{\Lambda^{c}}\left(\eta_{\Lambda^{c}}\right) \int_{\Gamma_{X} \times \Gamma_{X}} c\left(\eta_{\Lambda^{c}}+\pi^{\Lambda} \eta, \omega\right) \mathrm{d} \gamma\left(\eta, \omega \mid \eta_{\Lambda^{c}}\right) \\
& =\int_{\Gamma_{\Lambda^{c}}} \mathrm{~d} \mu_{\Lambda^{c}}\left(\eta_{\Lambda^{c}}\right) \int_{\Gamma_{\Lambda} \times \Gamma_{X}} c_{\eta_{\Lambda^{c}}}(\eta, \omega) \mathrm{d}\left(\pi^{\Lambda} \otimes \mathrm{Id}\right) \gamma\left(\eta, \omega \mid \eta_{\Lambda^{c}}\right) \\
& =J_{c_{\eta_{\Lambda^{c}}}}\left(\left(\pi^{\Lambda} \otimes \mathrm{Id}\right) \gamma\left(. \mid \eta_{\Lambda^{c}}\right)\right) .
\end{aligned}
$$

Now, note that $\left(\pi^{\Lambda} \otimes \mathrm{Id}\right) \gamma\left(. \mid \eta_{\Lambda^{c}}\right)$ has marginals $\pi^{\Lambda} \mu\left(. \mid \eta_{\Lambda^{c}}\right)$ and $p_{2} \gamma\left(. \mid \eta_{\Lambda^{c}}\right)$ which are probability measures on $\Gamma_{\Lambda}$ and $\Gamma_{X}$ respectively. Let $\mathcal{M}_{1}\left(\Gamma_{\Lambda} \times \Gamma_{X}\right)$ be the set of probability measures on $\Gamma_{\Lambda} \times \Gamma_{X}$. Define the sets $B$ and $C$ as

$$
\begin{gathered}
B=\left\{\left(\eta_{\Lambda^{c}}, \theta\right): \theta \in \Sigma\left(\pi^{\Lambda} \mu\left(. \mid \eta_{\Lambda^{c}}\right), p_{2} \gamma\left(. \mid \eta_{\Lambda^{c}}\right)\right\}\right. \\
C=\left\{\left(\eta_{\Lambda^{c}}, \theta\right): J_{c_{\eta_{\Lambda^{c}}}}(\theta)<J_{{c_{\eta_{\Lambda}}}}\left(\gamma\left(. \mid \eta_{\Lambda^{c}}\right)\right)\right\} .
\end{gathered}
$$

Let $K$ be the projection on $\Gamma_{\Lambda^{c}}$ of $C$. Since $B$ and $C$ are Borel, $K$ is a Souslin

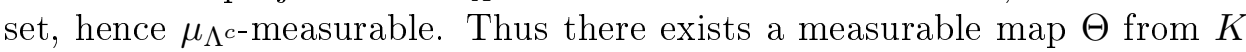
to $\mathcal{M}_{1}\left(\Gamma_{\Lambda} \times \Gamma_{X}\right)$ such that $\left(\eta_{\Lambda^{c}}, \Theta\left(\eta_{\Lambda^{c}}\right)\right)$ belongs to $C$, for $\mu_{\Lambda^{c} \text {-almost-all }}$ $\eta_{\Lambda^{c}}$. Define a measure $\theta$ as:

$$
\theta=\int_{K} \Theta\left(\eta_{\Lambda^{c}}\right) \mathrm{d} \mu_{\Lambda^{c}}\left(\eta_{\Lambda^{c}}\right)+\int_{K^{c}}\left(\pi^{\Lambda} \otimes \mathrm{Id}\right) \gamma\left(. \mid \eta_{\Lambda^{c}}\right) \mathrm{d} \mu_{\Lambda^{c}}\left(\eta_{\Lambda^{c}}\right) .
$$

If $\mu_{\Lambda^{c}}(K)>0$ then

$$
\begin{gathered}
J_{c}(\theta)=\int_{K^{c}} J_{c_{\eta_{\Lambda^{c}}}}\left(\left(\pi^{\Lambda} \otimes \mathrm{Id}\right) \gamma\left(. \mid \eta_{\Lambda^{c}}\right)\right) \mathrm{d} \mu_{\Lambda^{c}}\left(\eta_{\Lambda^{c}}\right) \\
+\int_{K} J_{c_{\eta_{\Lambda^{c}}}}\left(\Theta\left(\eta_{\Lambda^{c}}\right)\right) \mathrm{d} \mu_{\Lambda^{c}}\left(\eta_{\Lambda^{c}}\right) \\
<\int_{K^{c}} J_{c_{\eta_{\Lambda^{c}}}}\left(\left(\pi^{\Lambda} \otimes \mathrm{Id}\right) \gamma\left(. \mid \eta_{\Lambda^{c}}\right)\right) \mathrm{d} \mu_{\Lambda^{c}}\left(\eta_{\Lambda^{c}}\right) \\
+\int_{K} J_{c_{\eta_{\Lambda^{c}}}}\left(\left(\pi^{\Lambda} \otimes \mathrm{Id}\right) \gamma\left(. \mid \eta_{\Lambda^{c}}\right)\right) \mathrm{d} \mu_{\Lambda^{c}}\left(\eta_{\Lambda^{c}}\right) \\
=J_{c}(\gamma),
\end{gathered}
$$

which is a contradiction to the optimality of $\gamma$.

Theorem 5.1. Assume that the hypothesis of Lemma 5.1 holds and assume that $\mu$ is regular. Let $\Lambda$ be any compact subset of $X$. Then, there exists a measurable map $T_{\Lambda}$ from $\Gamma_{X}$ to itself such that

$$
\gamma\left((\eta, \omega): r_{2}^{\Lambda}\left(\left\{\beta_{\eta, \omega}\right\}\right)=T_{\Lambda}\left(\pi^{\Lambda} \eta, \pi^{\Lambda^{c}} \eta\right)\right)=1
$$

Proof. Fix $\eta_{\Lambda^{c}} \in \Gamma_{\Lambda^{c}}$ and define $C_{\eta_{\Lambda^{c}}}$ as the support of $\gamma\left(. \mid \eta_{\Lambda^{c}}\right)$. Consider the two sets:

$$
\left.K_{\eta_{\Lambda^{c}}}=\left\{\left(\eta, r_{2}^{\Lambda}\left(\left\{\beta_{\eta+\eta_{\Lambda^{c}}}, \omega\right\}\right)\right) \in \Gamma_{\Lambda} \times \Gamma_{X}:\left(\eta+\eta_{\Lambda^{c}}, \omega\right\}\right) \in C_{\eta_{\Lambda^{c}}}\right\}
$$

and

$$
K_{\eta_{\Lambda^{c}}, \eta}=\left\{\omega \in \Gamma_{X},(\eta, \omega) \in K_{\eta_{\Lambda^{c}}}\right\}
$$




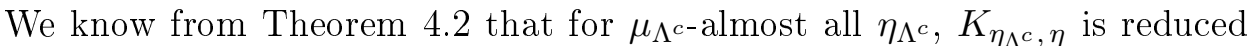
to one point for $\mu_{\Lambda}\left(. \mid \eta_{\Lambda^{c}}\right)$-almost-all $\eta$. Let

$$
N=\left\{\left(\eta, \eta_{\Lambda^{c}}\right) \in \Gamma_{\Lambda} \times \Gamma_{\Lambda^{c}}: \quad \operatorname{Card}\left(K_{\eta, \eta_{\Lambda^{c}}}\right)>1\right\}
$$

$N$ is a Souslin set, hence it is universally measurable. Let $\sigma$ be the measure defined as the image of $\mu$ under the projection $\eta \mapsto\left(\pi^{\Lambda} \eta, \pi^{\Lambda^{c}} \eta\right)$. We then have

$$
\sigma(N)=\int_{\Gamma_{\Lambda^{c}}} \mathrm{~d} \mu_{\Lambda^{c}}\left(\eta_{\Lambda^{c}}\right) \int_{\Gamma_{\Lambda}} \mathbf{1}_{N}\left(\eta, \eta_{\Lambda^{c}}\right) \mu\left(\mathrm{d} \eta \mid \eta_{\Lambda^{c}}\right)=0
$$

Hence, $\mu$ and $\gamma$ almost-surely, $K_{\eta_{\Lambda^{c}}, \eta}$ is reduced to a single-point and we define $T_{\Lambda}$ as the map which sends $\left(\eta, \eta_{\Lambda^{c}}\right)$ to this point. It is automatically measurable by the selection theorem.

Theorem 5.2. Assume that the hypothesis of Lemma 5.1 holds and assume that $\mu$ is regular. Let $\left(\Lambda_{n}, n \geq 1\right)$ be an increasing sequence of compact sets such that $\cup_{n \geq 1} \Lambda_{n}=X$. Then, there exists a unique optimal measure $\gamma$ and a unique map $T$ such that

$$
\gamma=(\operatorname{Id} \otimes T)^{*} \mu
$$

Proof. Let $\gamma$ be an optimal measure for $\operatorname{MKP}(\mu, \nu, c)$. According to Theorem 5.1, we know that

$$
r_{2}^{\Lambda_{n}}\left(\left\{\beta_{\eta, \omega}\right\}\right)=T_{\Lambda_{n}}\left(\pi^{\Lambda_{n}} \eta, \pi^{\Lambda_{n}^{c}} \eta\right)
$$

$\gamma$-a.s. for all integers $n$. Let $B$ be a bounded subset of $X$, we clearly have

$$
r_{2}^{\Lambda_{n}}\left(\beta_{\eta, \omega}\right)(B) \leq \omega(B)<\infty,
$$

for any $\beta_{\eta, \omega}$ realizing $c(\eta, \omega)$. Thus, for $\gamma$-almost all $(\eta, \omega)$, the family $\left(r_{2}^{\Lambda_{n}}\left(\left\{\beta_{\eta, \omega}\right\}\right), n \geq 1\right)$ is tight in $\Gamma_{X}$ (see [Kal83]). Hence, up to the extraction of a subsequence, one can assume that $r_{2}^{\Lambda_{n}}\left(\left\{\beta_{\eta, \omega}\right\}\right)$ converges to $\omega$. On the other hand, $\pi^{\Lambda_{n}} \eta$ converges to $\eta$ and $\pi^{\Lambda_{n}^{c}} \eta$ converges to $\emptyset$ as $n$ goes to infinity. Define $T$ by $T(\eta)=\lim _{n \rightarrow \infty} T_{\Lambda_{n}}\left(\pi^{\Lambda_{n}} \eta, \pi^{\Lambda_{n}^{c}} \eta\right)$, we clearly have $\omega=T(\eta)$, $\gamma$-a.s. The conclusion follows by Theorem 2.1

We didn't manage to find any sufficient condition which would ensure the finiteness of the Wasserstein distance between two locally finite point processes. However, we do know that there exists some relevant cases. Consider, for instance, we are given a Poisson process of non-finite intensity $\sigma_{1}$ and a map $h$ from $X$ to itself such that $\int\|h\|^{2} \mathrm{~d} \sigma_{1}$ is finite. Then,

$$
\begin{aligned}
\mathcal{T}_{c}\left(\mu_{\sigma_{1}},\left(\operatorname{Id}+h^{\Gamma}\right)^{*} \mu_{\sigma_{1}}\right) & \leq \frac{1}{2} \mathbf{E}\left[\sum_{x \in \eta}\|x-(\operatorname{Id}+h)(x)\|^{2} \mathrm{~d} \eta(x)\right] \\
& =\frac{1}{2} \int\|h\|^{2} \mathrm{~d} \sigma_{1}<\infty .
\end{aligned}
$$

Note that $\left(\operatorname{Id}+h^{\Gamma}\right)^{*} \mu_{\sigma_{1}}$ is a Poisson process of intensity $(\operatorname{Id}+h)^{*} \sigma_{1}$. 


\section{REFERENCES}

[AKR98] S. Albeverio, Yu. G. Kondratiev, and M. Rockner, Analysis and geometry on configuration spaces, J. Funct. Anal. 154 (1998), no. 2, 444-500. MR 99

[BB92] A. D. Barbour and T. C. Brown, Stein's method and point process approximation, Stochastic Process. Appl. 43 (1992), no. 1, 9-31. MR MR1190904 (93k:60120)

[BC01] A. D. Barbour and O. Chryssaphinou, Compound Poisson approximation: a user's guide, Ann. Appl. Probab. 11 (2001), no. 3, 964-1002. MR 2002

[BHJ92] A. D. Barbour, L. Holst, and S. Janson, Poisson approximation, Oxford Studies in Probability, vol. 2, The Clarendon Press Oxford University Press, 1992, Oxford Science Publications. MR 93

[BM02] A. D. Barbour and M. Maansson, Compound Poisson process approximation, Ann. Probab. 30 (2002), no. 3, 1492-1537. MR 1

[BX00] A. D. Barbour and A. Xia, Estimating Stein's constants for compound Poisson approximation, Bernoulli 6 (2000), no. 4, 581-590. MR 2001

[DVJ03] D. J. Daley and D. Vere-Jones, An introduction to the theory of point processes. Vol. I, second ed., Probability and its Applications (New York), SpringerVerlag, New York, 2003, Elementary theory and methods. MR MR1950431 (2004c:60001)

[FÜ04] D. Feyel and A. S. Üstünel, Monge-Kantorovitch measure transportation and Monge-Ampère equation on Wiener space, Probab. Theory Related Fields 128 (2004), no. 3, 347-385. MR MR2036490 (2004m:60121)

[Kal83] O. Kallenberg, Random measures, 3rd ed., Academic Press, 1983.

[Lev99] V. Levin, Abstract cyclical monotonicity and Monge solutions for the general Monge-Kantorovich problem, Set-Valued Anal. 7 (1999), no. 1, 7-32. MR MR1699061 (2000j:90075)

[RR98a] S. T. Rachev and L. Rüschendorf, Mass transportation problems. Vol. I, Probability and its Applications (New York), Springer-Verlag, New York, 1998, Theory. MR 99

[RR98b] , Mass transportation problems. Vol. II, Probability and its Applications (New York), Springer-Verlag, New York, 1998, Applications. MR 99

[RS99] M. Röckner and A. Schied, Rademacher's theorem on configuration spaces and applications, J. Funct. Anal. 169 (1999), no. 2, 325-356. MR MR1730565 (2001b:58058)

[Rüs96] L. Rüschendorf, On c-optimal random variables, Statist. Probab. Lett. 27 (1996), no. 3, 267-270. MR MR1395577 (97h:62051)

[Tho00] H. Thorisson, Coupling, stationarity, and regeneration, Probability and its Applications (New York), Springer-Verlag, New York, 2000, pp. xiv +517.

[Vi103] C. Villani, Topics in optimal transportation, Graduate Studies in Mathematics, vol. 58, American Mathematical Society, Providence, RI, 2003. MR MR1964483 (2004e:90003)

[Xia00] A. Xia, Poisson approximation, compensators and coupling, Stochastic Anal. Appl. 18 (2000), no. 1, 159-177. MR 2001

L. Decreusefond, GeT/ENST - UMR CNRS 5141, 46, rue Barrault, 75634

PARIS CEDEX 13, FRANCE

E-mail address | : Laurent.Decreusefond@enst.fr 